\title{
Uso seguro de medicamentos na atenção primária à saúde no Brasil: revisão integrativa
}

\section{Safe use of medicines in the context of primary health care in Brazil: integrative review}

\author{
Rodrigo Fonseca Lima' • Alexandre Vaz Machado ${ }^{2} \bullet$ Maria Luiza Rêgo Bezerra ${ }^{3}$
}

\begin{abstract}
RESUMO
Objetivo: Identificar, descrever e comparar estudos relacionados à temática de uso seguro de medicamentos no âmbito da APS no Brasil. Métodos: Revisão integrativa realizada através de levantamento bibliográfico relacionado ao uso seguro de medicamentos no contexto da APS no Brasil nas bases SciELO, LILACS, MEDLINE, IBECS, PubMed, PubMed Central, SCOPUS, Science Direct e Web of Science entre 2008 e 2018 com subsequente avaliação de nível de evidência. Resultados: Dos seis artigos da amostra final, cinco eram do tipo transversal (nível de evidência 6) e os aspectos relacionados à segurança do paciente mais abordados foram interações medicamentosas potenciais $(n=3 ; 50 \%)$ e erros de prescrição $(n=3 ; 50 \%)$. Conclusão: Apesar de serem observadas sugestões importantes com objetivo de melhorar o processo de uso de medicamentos na APS, a abordagem da temática na literatura científica brasileira é escassa e com baixo nível de evidência associado considerando sua importância em termos de assistência à saúde.

Descritores: Atenção Primária à Saúde; Segurança do Paciente; Uso de Medicamentos; Brasil; Revisão.
\end{abstract}

\begin{abstract}
Objective:To identify, describe and compare studies related to the topic of safe use of medicines in the context of PHC in Brazil. Methods: Integrative review carried out through a bibliographic survey related to the safe use of medicines in the context of PHC in Brazil based on SciELO, LILACS, MEDLINE, IBECS, PubMed, PubMed Central, SCOPUS, Science Direct and Web of Science between 2008 and 2018 with subsequent level of evidence assessment. Results: Of the six articles in the final sample, five were cross-sectional (level of evidence 6) and the aspects most addressed to patient safety were potential drug interactions $(n=3 ; 50 \%)$ and prescription errors $(n=3 ; 50 \%)$. Conclusion:Although important suggestions are observed in order to improve the process of using medicines in PHC, the approach to the topic in Brazilian scientific literature is scarce and with a low level of associated evidence considering its importance in terms of health care.
\end{abstract}

Descriptors: Primary Health Care; Patient Safety; Drug Utilization; Brazil; Review.

I Doutor em Ciências Farmacêuticas pela Universidade de Brasília (2018), mestre em Ciências pelo Centro de Pesquisas Aggeu Magalhães/FIOCRUZ/PE (20I3) (área de concentração: Epidemiologia, Políticas e Gestão em Saúde/ Avaliação de serviços de saúde) e especialista em Farmacologia Clínica (IBPEx/2013), em Farmácia Hospitalar por concurso público (prova e títulos) (SBRAFH/2015) e em Farmácia Clínica e Hospitalar (UNINTER/2019).Atualmente é farmacêutico hospitalar da Secretaria de Saúde do Distrito Federal e docente na Universidade de Brasília (professor substituto) e desenvolve pesquisa relacionada a serviços farmacêuticos hospitalares gerenciais e assistenciais com foco especial na temática de Segurança do Paciente.

2 Farmacêutico Clínico e Industrial pela Universidade de Brasília, pós graduado em farmacologia clínica pelo Centro de Pós Graduação Oswaldo Cruz e mestre em Ciências Farmacêuticas pela Universidade de Brasília. Farmacêutico da Secretária de Saúde do Distrito Federal e preceptor do Programa de Residência Multiprofissional em Saúde da Família e Comunidade da Escola Superior de Ciências da Saúde (ESCS).

3 Doutoranda em Bioética (UnB). Mestre em Enfermagem (UnB). Especialista em Gestão em Saúde Pública com ênfase em Saúde da Família (INESPO). Enfermeira (UFMA). Docente do Curso de Enfermagem da Universidade Paulista (Unip), campus Brasilia. 


\section{INTRODUÇÃO}

A Atenção Primária à Saúde (APS) é viabilizada no Brasil no contexto do Sistema Único de Saúde (SUS) através da Estratégia Saúde da Família no âmbito das Redes de Atenção à Saúde (RAS). Corresponde ao primeiro contato com serviços de saúde e envolve ações de prevenção, promoção da saúde, reabilitação, redução de risco ou manutenção de baixo risco, detecção precoce, rastreamento e tratamento de doenças com ênfase nas ações interdisciplinares em saúde voltadas para a população adscrita mediante uso de métodos e tecnologias em saúde considerando os princípios doutrinários e organizacionais do SUS ${ }^{(1-3)}$.

Cuidados primários à saúde apresentam riscos inerentes de ocorrência de eventos adversos, seja por conta do ambiente onde são realizados quanto por conta do tipo de ação em saúde realizada. Nesse sentido, a discussão acerca da temática de segurança do paciente deve tomar proporções cada vez mais relevantes nesse contexto na intenção de prevenção de eventos adversos e melhora das atividades assistenciais em saúde ${ }^{(4-5)}$.

Os eventos adversos podem estar associados ao diagnóstico, ao tratamento medicamentoso e a aspectos organizacionais, envolvendo, por exemplo, problemas de comunicação efetiva entre membros da equipe interdisciplinar em saúde e ambiente de trabalho ${ }^{(6-7)}$. A literatura aponta que, dentre os eventos acima mencionados, os relacionados ao uso de medicamentos (desde a prescrição até a dispensação) são os mais frequentes ${ }^{(4 ; 6)}$.

Ratifica-se que a melhora na comunicação entre os profissionais de saúde é fator crucial para a otimização da assistência com vistas na redução de eventos adversos relacionados à segurança do paciente, com ênfase nos mais frequentes, a saber, relacionados ao uso, administração e prescrição de medicamentos e que neste universo encontram-se as ações de enfermeiros, farmacêutico e médicos como essenciais e preponderantes ao processo ${ }^{(4-5,8-10)}$.

O processo de uso de medicamentos, especificamente, é passível de falhas que podem tanto causar dano direto como privar o usuário do benefício terapêutico e corresponde a um dos domínios no contexto das estratégias e ações para gestão de risco no âmbito das organizações de saúde envolvendo serviços inter-relacionados em um processo complexo e interdisciplinar ${ }^{(10-14)}$.

O contexto desfavorável à segurança do paciente embasou a elaboração de metas internacionais relacionadas à temática que envolvem aspectos como identificação correta dos pacientes e o uso seguro de medicamentos, cujo cumprimento perpassa necessariamente por aspectos intersetorais que devem ser constantemente monitorados com vistas à melhoria da qualidade e segurança de suas ações ${ }^{(1-12,15-18)}$. Independentemente do âmbito onde essas ações de saúde são realizadas, há uma grande preocupação no que se refere à cultura de segurança, atributo incorporado à qualidade da assistência à saúde que remete ao compromisso de uma organização de saúde quanto à gestão da segurança do paciente ${ }^{(16,19-21)}$.

Os serviços previstos na APS correspondem a um dos primeiros pontos de contato de pacientes com o sistema de saúde e tem se tornado cada vez mais complexos decorrentes da ampliação de demandas clínicas que envolvem os diversos atores da equipe interdisciplinar que devem trabalhar de forma conjunta com vistas à integralidade das ações ${ }^{(5,22)}$. É nesse contexto que se justifica a crescente discussão interdisciplinar acerca da segurança do paciente no âmbito da assistência prestada na atenção primária, a qual também tem riscos inerentes que podem estar relacionados ao diagnóstico, à estrutura organizacional e ao tratamento, sendo esse último o mais associado a eventos adversos. Tal discussão, no entanto, ainda se encontra incipiente no âmbito da APS ${ }^{(5,23)}$.

Desta forma, tem-se como objetivo desta pesquisa, identificar, descrever e comparar estudos relacionados à temática de uso seguro de medicamentos no âmbito da APS no Brasil.

\section{MÉTODO}

Trata-se de uma revisão integrativa realizada por meio de levantamento bibliográfico relacionado ao uso seguro de medicamentos no contexto da APS no Brasil realizada com o objetivo de síntese de resultados mediante metodologia padronizada ${ }^{(24-25)}$.

Com o intuito de responder a pergunta norteadora: "Quais aspectos quali-quantitativos acerca da temática de uso seguro de medicamentos na APS foram evidenciados na literatura no período de 2008 a 2018 no Brasil?", foi realizado levantamento dos artigos na literatura a partir das bases de dados das ciências da saúde em geral SciELO (Scientific Electronic Library Online), LILACS (Literatura Latino-Americana e do Caribe), MEDLINE (National Library of Medicine, Estados Unidos), IBECS (Indice Bibliográfico Español de Ciencias de la Salud, Espanha), PubMed (US National Library of Medicine National Institutes of Health), PubMed Central (PubMed C), SciVerse SCOPUS, Science Direct e Web of Science.

A busca eletrônica foi realizada por meio da combinação dos Descritores em Ciências da Saúde (DeCS) (em inglês): family practice (Medicina de Família e Comunidade) OU primary care (Atenção Primária à Saúde) OU primary health care (Atenção Primária à Saúde) OU general practice (Medicina Geral) E patient safety (Segurança do Paciente) E drug utilization (Uso de Medicamentos) OU medical error (Erros médicos) OU medication error (Erros de medicação) OU adverse drug reaction (Efeitos Colaterais e Reações Adversas Relacionados a Medicamentos) 
AND Brazil (Brasil) e se baseou na adoção do critério de inclusão referente à indexação de artigos em bases de dados sem limitação de idioma com tema central e discussão relacionados uso seguro de medicamentos na APS e publicados de 2008 até dezembro de 2018 . Foram definidos como critérios de exclusão: cartas ao editor, artigos de opinião, produções sem disponibilidade do texto na íntegra, revisões, relatos de caso, capítulos de livro, resumos de eventos, estudos envolvendo serviços em nível de atendimento hospitalar, especializado ou em centros de referência, estudos envolvendo processos e/ ou enfermidades e/ou medicamentos específicos na APS.

O recorte temporal se deveu ao fato de que em 2008 foi publicada uma revisão sistemática viabilizada pela Organização Mundial da Saúde (OMS) que teve como objetivo levantar na literatura as metodologias disponíveis para realização de pesquisas sobre segurança do paciente na atenção primária à saúde até dezembro de 2007 utilizando como estratégia de busca os descritores específicos ${ }^{(4)}$ (nessa revisão publicada em 2008 não houve especificação quanto ao tema de uso seguro de medicamentos).

Uma primeira análise foi realizada pela leitura dos títulos das publicações, seguida pela leitura e análise crítica dos resumos respeitando os critérios de exclusão. Em seguida, foi realizada a verificação dos artigos na íntegra para identificação das ideias centrais. A busca inicial para a seleção dos títulos dos artigos foi realizada por dois autores de forma independente cabendo ao terceiro autor a definição de inserção ou não de um estudo na amostra final em caso de dúvida.

Os dados foram coletados por meio de instrumento

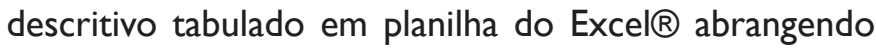
procedência, título, autoria, periódico, metodologia, objetivos, resultados e conclusão. As referências foram ainda analisadas conforme ano, idioma e local de publicação, aspectos específicos relacionados à segurança do paciente abordados e sugestões/ propostas de intervenções.

Para a avaliação do nível de evidência dos estudos foi utilizada a escala Rating System for the Hierarchy of Evidence for Intervention/ Treatment Questions ${ }^{(26)}$, sendo considerados somente os níveis 2, 3, 4 e 6 (evidências de ensaios clínicos randomizados, de ensaios clínicos sem randomização, de caso-controle e estudos de coorte e de estudos descritivos ou qualitativos, respectivamente); os demais níveis não foram considerados por corresponderem a tipos de publicação pertencentes aos critérios de exclusão da presente revisão (nível I: revisões sistemáticas ou meta-análise de ensaios clínicos randomizados; nível 5: revisões sistemáticas de estudos descritivos e qualitativos; e nível 7: evidências de opinião de autoridades/relatórios de experts).

Descritores: family practice (Medicina de Familia e Comunidade) OU primary care (Atenção Primária à Saúde) OU primarxy health care (Atenção Primária à Saúde) OU general practice (Medicina Geral) E patient safețu (Segurança do Paciente) E drug. utilization (Uso de Medicamentos) OU medical error. (Erros médicos) OU medication exror (Erros de medicação) OU adverse drug reaction (Efeitos Colaterais e Reações Adversas Relacionados a Medicamentos) AND Brazill(Brasil)

\begin{tabular}{|l|c|}
\hline \multicolumn{1}{|c|}{} & Recorte temporal (2008-2018) e de disponibilidade (definição das referências elegiveis) \\
\cline { 2 - 3 } & 638 artigos \\
\cline { 2 - 3 } & Análise de títulos e resumos (definição das referências para leitura na integra) e exclusão \\
\hline & de referências repetidas \\
\cline { 2 - 2 } & 17 artigos \\
\hline
\end{tabular}

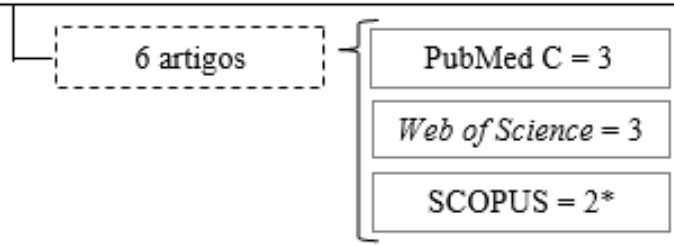

FIGURA 1 - Representação esquemática da obtenção da amostra. Brasília-DF, Brasil, 2018. Fonte: Os autores (2018) 


\section{RESULTADOS}

A amostra final para análise se constituiu em seis artigos do total de 77I disponíveis após busca avançada com os descritores (figura I).

Todos os seis artigos da amostra final estavam em língua inglesa e/ou portuguesa e quatro deles $(66,7 \%)$ foram realizados nas regiões sul e sudeste (dos demais, um foi realizado na região nordeste e o outro envolveu dados de todas as regiões brasileiras). $O$ tipo de estudo mais frequente foi o transversal $(n=5 ; 83,3 \%)$ (somente um estudo apresentou metodologia prospectiva ( $16,7 \%)$, com nível de evidência mais forte - nível 3) e os aspectos relacionados à segurança do paciente mais abordados foram os associados a interações medicamentosas potenciais (IMP) $(n=3 ; 50 \%)$, erros de prescrição $(n=3 ; 50 \%)$ e polifarmácia $(n=2 ; 33,3 \%)$.A análise quali-quantitativa das referências e a caracterização da abordagem, principais resultados e conclusões da amostra final estão apresentados nos quadros I e 2.

QUADRO 1 - Análise quali-quantitativa dos artigos da amostra final. Brasília-DF, Brasil, 2018.

\begin{tabular}{|c|c|c|c|c|c|}
\hline Autores & Ano & Objetivos & $\begin{array}{c}\text { Local do } \\
\text { estudo }\end{array}$ & Metodologia & $\begin{array}{c}\text { Nível de } \\
\text { evidência }\end{array}$ \\
\hline $\begin{array}{l}\text { Nicolini P, Nascimento } \\
\text { JWL, Greco KV, } \\
\text { Menezes FG }\end{array}$ & 2008 & $\begin{array}{c}\text { Analisar prescrições } \\
\text { contendo antibióticos } \\
\text { quanto aos padrões de } \\
\text { uso e IMP }\end{array}$ & São Paulo & $\begin{array}{l}\text { Estudo do tipo transversal em que foi aplicado } \\
\text { questionário sobre o uso de antibióticos. } \\
\text { Foram consideradas as variáveis: faixa etária, } \\
\text { prescritor (especialidade médica), número de } \\
\text { medicamentos prescritos, conhecimento do } \\
\text { paciente com relação à doença e à posologia, } \\
\text { número de prescrições com nome genérico e } \\
\text { IMP }\end{array}$ & 6 \\
\hline $\begin{array}{l}\text { Mendonca JMD, Lyra } \\
\text { Junior DP, Rabelo JS, } \\
\text { Siqueira, JS, Balisa- } \\
\text { Rocha BJ, Gimenes } \\
\text { FRE, et al. }{ }^{(28)}\end{array}$ & 2010 & $\begin{array}{c}\text { Analisar erros } \\
\text { de prescrições } \\
\text { odontológicas com } \\
\text { vistas a sugestões } \\
\text { de melhorias viáveis } \\
\text { para a segurança do } \\
\text { paciente }\end{array}$ & Sergipe & $\begin{array}{c}\text { Estudo transversal descritivo de cunho } \\
\text { avaliativo utilizando como base indicadores } \\
\text { padronizados para avaliação de prescrições } \\
\text { odontológicas }\end{array}$ & 6 \\
\hline $\begin{array}{l}\text { Teixeira JJV, Crozatti } \\
\text { MTL, Dos Santos CA, } \\
\text { Romano-Lieber NS }\end{array}$ & 2012 & $\begin{array}{l}\text { Investigar a } \\
\text { prevalência de IMP em } \\
\text { pacientes atendidos } \\
\text { na APS }\end{array}$ & Paraná & $\begin{array}{c}\text { Estudo transversal realizado com pacientes } \\
\text { de idade igual ou superior a } 45 \text { anos. Dados } \\
\text { coletados a partir de prescrições apresentadas } \\
\text { para dispensação do medicamento ao paciente } \\
\text { (os prontuários eletrônicos dos pacientes } \\
\text { foram conferidos) }\end{array}$ & 6 \\
\hline $\begin{array}{l}\text { Marchon SG, Mendes } \\
\text { Junior WV, Pavão } \\
\text { AL }^{(30)}\end{array}$ & 2015 & $\begin{array}{l}\text { Avaliar a ocorrência de } \\
\text { incidentes no cuidado } \\
\text { à saúde ao paciente }\end{array}$ & $\begin{array}{l}\text { Rio de } \\
\text { Janeiro }\end{array}$ & $\begin{array}{l}\text { Estudo observacional prospectivo. Quinze } \\
\text { profissionais de saúde registraram de forma } \\
\text { anônima e confidencial, durante cinco meses, } \\
\text { incidentes ocorridos (erros no atendimento } \\
\text { administrativo; erros na investigação de } \\
\text { exames; erros com tratamento; erros de } \\
\text { comunicação; erros de pagamento; erros } \\
\text { na gestão de recursos humanos; erros na } \\
\text { execução de uma tarefa clínica; erros de } \\
\text { diagnóstico) com os pacientes durante cinco } \\
\text { meses, através do questionário Primary Care } \\
\text { International Study of Medical Errors (PCISME) } \\
\text { adaptado para o Brasil. }\end{array}$ & 3 \\
\hline $\begin{array}{l}\text { Cruz HL, Mota FKC, } \\
\text { Araújo LU, Bodevan } \\
\text { EC, Seixas SRS, } \\
\text { Santos DF(31) }\end{array}$ & 2017 & $\begin{array}{l}\text { Descrever a história de } \\
\text { uso de medicamentos } \\
\text { a partir dos prontuários } \\
\text { para medir fatores } \\
\text { associados aos } \\
\text { erros relacionados } \\
\text { ao processo de uso } \\
\text { de medicamentos } \\
\text { em pacientes com } \\
\text { doenças crônicas }\end{array}$ & $\begin{array}{l}\text { Minas } \\
\text { Gerais }\end{array}$ & $\begin{array}{l}\text { Estudo transversal retrospectivo envolvendo } \\
\text { dados de prontuários. Os erros relacionados } \\
\text { ao processo de uso de medicamentos foram } \\
\text { classificados de acordo com os danos que o } \\
\text { medicamento poderia causar ao paciente }\end{array}$ & 6 \\
\hline $\begin{array}{l}\text { Nascimento RCRM, } \\
\text { Álvares J, Guerra } \\
\text { Junior AA, Gomes IC, } \\
\text { Silveira MR, Costa EA, } \\
\quad \text { et al. }{ }^{(32)}\end{array}$ & 2017 & $\begin{array}{l}\text { Caracterizar a } \\
\text { polifarmácia em } \\
\text { usuários da APS e } \\
\text { identificar fatores a ela } \\
\text { associados (variáveis } \\
\text { sociodemográficas } \\
\text { e indicadores de } \\
\text { condições de saúde) }\end{array}$ & $\begin{array}{l}\text { Diversos } \\
\text { municípios } \\
\text { brasileiros }\end{array}$ & $\begin{array}{l}\text { Estudo transversal, exploratório, de cunho } \\
\text { avaliativo integrante do Componente } \\
\text { Serviços da Pesquisa Nacional sobre Acesso, } \\
\text { Utilização e Promoção do Uso Racional de } \\
\text { Medicamentos (2015) }\end{array}$ & 6 \\
\hline
\end{tabular}




\begin{tabular}{|c|c|c|c|}
\hline Autores & $\begin{array}{c}\text { Aspectos relacionados } \\
\text { à segurança no uso } \\
\text { de medicamentos } \\
\text { abordados }\end{array}$ & Principais resultados & $\begin{array}{c}\text { Intervenções propostas e } \\
\text { conclusões }\end{array}$ \\
\hline $\begin{array}{c}\text { Nicolini P, Nascimento } \\
\text { JWL, Greco KV, Menezes } \\
\text { FG }^{(27)}\end{array}$ & IMP, erros de prescrição & $\begin{array}{l}\text { Foram entrevistados } 149 \text { pacientes; } 10,7 \% \\
\text { desconheciam o diagnóstico da sua doença } \\
\text { e } 15,4 \% \text { não entendiam a posologia do } \\
\text { antibiótico. Em } 8,0 \% \text { dos casos houve } \\
\text { IMP; } 42,9 \% \text { delas foram consideradas de } \\
\text { gravidade moderada, não sendo observadas } \\
\text { interações de alta gravidade }\end{array}$ & $\begin{array}{l}\text { Segundo os autores, são } \\
\text { necessárias intervenções como } \\
\text { treinamento da equipe envolvida } \\
\text { para comunicação efetiva e } \\
\text { atividades de educação em saúde } \\
\text { para viabilizar a qualidade e } \\
\text { segurança da assistência no que } \\
\text { se refere ao uso de medicamentos }\end{array}$ \\
\hline $\begin{array}{l}\text { Mendonca JMD, Lyra } \\
\text { Junior DP, Rabelo JS, } \\
\text { Siqueira, JS, Balisa-Rocha } \\
\text { BJ, Gimenes FRE, et al. }{ }^{(28)}\end{array}$ & Erros de prescrição & $\begin{array}{c}\text { Foram avaliadas } 297 \text { prescrições; } 98,3 \% \\
\text { delas continham abreviaturas e } 26 \% \text { foram } \\
\text { classificadas como de baixa legibilidade ou } \\
\text { ilegíveis. As inadequações e/ou omissões } \\
\text { de dosagem }(67,0 \%) \text { e administração } \\
(58,9 \%) \text { dos medicamentos foram as } \\
\text { mais frequentes; } 95,5 \% \text { das prescrições } \\
\text { não apresentavam ou apresentavam de } \\
\text { forma inadequada orientações de uso dos } \\
\text { medicamentos }\end{array}$ & $\begin{array}{l}\text { Conforme os autores, potenciais } \\
\text { erros de prescrição devem ser } \\
\text { avaliados com vistas à melhoria e } \\
\text { segurança no processo de uso de } \\
\text { medicamentos e, nesse contexto, } \\
\text { a equipe interdisciplinar tem papel } \\
\text { fundamental }\end{array}$ \\
\hline $\begin{array}{c}\text { Teixeira JJV, Crozatti MTL, } \\
\text { Dos Santos CA, Romano- } \\
\text { Lieber NS }\end{array}$ & IMP & $\begin{array}{l}\text { Foram investigados } 827 \text { pacientes (média } \\
\text { de medicamentos: } 4,4 \text { ). A prevalência IMP } \\
\text { foi de } 63,0 \% \text {, sendo } 12,1 \% \text { de gravidade } \\
\text { severa. O número de medicamentos } \\
\text { prescritos foi significativamente associado } \\
\text { a IMP, com risco crescente conforme maior } \\
\text { número de medicamentos em uso }\end{array}$ & $\begin{array}{l}\text { Os autores chamam atenção } \\
\text { para conscientização quanto à } \\
\text { prescrição tendo em vista que um } \\
\text { número maior de medicamentos } \\
\text { prescritos foi fator preditor para } \\
\text { IMP. Referem ainda a importância } \\
\text { de uma lista de medicamentos } \\
\text { que ofereça base para prescrição } \\
\text { e dispensação de medicamentos } \\
\text { seguros aos pacientes }\end{array}$ \\
\hline $\begin{array}{l}\text { Marchon SG, Mendes } \\
\text { Junior WV, Pavão AL }\end{array}$ & $\begin{array}{l}\text { Erros no atendimento } \\
\text { administrativo*, erros de } \\
\text { prescrição }\end{array}$ & $\begin{array}{l}\text { Foram registrados } 125 \text { incidentes em } \\
11233 \text { consultas. A taxa de incidência } \\
\text { geral foi de } 1,1 \% \text {. A taxa de incidência de } \\
\text { incidentes que atingiram os pacientes, mas } \\
\text { não causaram danos e a de incidentes } \\
\text { que causaram evento adverso foi de } 0,1 \% \\
\text { e } 0,9 \% \text {, respectivamente. O tipo de erro } \\
\text { relacionado ao tratamento foi o terceiro mais } \\
\text { frequente }(n=24 ; 19 \%) \text {, atrás dos erros de } \\
\text { cunho administrativo }(n=32 ; 26 \%) \text { e erros } \\
\text { de comunicação ( } n=27 ; 22 \%) ; 10,0 \% \text { dos } \\
\text { erros estavam relacionados ao serviço } \\
\text { farmacêutico }\end{array}$ & $\begin{array}{l}\text { As taxas de incidência } \\
\text { remetem, segundo os autores, } \\
\text { à necessidade de estudos } \\
\text { no contexto da segurança do } \\
\text { paciente no âmbito da APS }\end{array}$ \\
\hline $\begin{array}{c}\text { Cruz HL, Mota FKC, } \\
\text { Araújo LU, Bodevan EC, } \\
\text { Seixas SRS, Santos DF(31) }\end{array}$ & $\begin{array}{l}\text { IMP, polifarmácia, uso } \\
\text { de medicamento de } \\
\text { alto risco (risco de } \\
\text { queda, baixa margem } \\
\text { terapêutica, risco } \\
\text { renal), medicamentos } \\
\text { inapropriados e terapia } \\
\text { duplicada }\end{array}$ & $\begin{array}{c}396 \text { prontuários de pacientes com } \\
\text { diabetes e hipertensão foram analisados. } \\
\text { A polifarmácia foi registrada em } 54 \% \text { da } \\
\text { amostra; } 67,0 \% \text { dos medicamentos incluíam } \\
\text { pelo menos um risco. Os riscos mais } \\
\text { comuns foram: IMP }(57,8 \%), \text { risco renal } \\
(29,8 \%) \text {, risco de queda }(12,9 \%) \text { e terapias } \\
\text { duplicadas }(11,9 \%)\end{array}$ & $\begin{array}{l}\text { Conforme autores, os fatores } \\
\text { relacionados precisam ser } \\
\text { tratados individualmente em } \\
\text { todos os serviços de saúde, com } \\
\text { atenção especial ao histórico de } \\
\text { uso de medicamentos, sendo } \\
\text { importante a reconciliação nos } \\
\text { pontos de entrada ao sistema de } \\
\text { saúde }\end{array}$ \\
\hline $\begin{array}{l}\text { Nascimento RCRM, } \\
\text { Álvares J, Guerra Junior } \\
\text { AA, Gomes IC, Silveira } \\
\text { MR, Costa EA, et al. }{ }^{(32)}\end{array}$ & Polifarmácia & $\begin{array}{c}\text { A prevalência de polifarmácia foi de } 9,4 \% \\
\text { na população geral (amostra de } 9000 \\
\text { usuários) ( } 18,1 \% \text { em idosos acima de } 65 \\
\text { anos). Houve associação entre polifarmácia } \\
\text { e faixa etária acima de } 45 \text { anos, baixa } \\
\text { autopercepção de saúde, presença de } \\
\text { doenças crônicas, ter plano de saúde, } \\
\text { atendimento em serviço de emergência e } \\
\text { região do país. }\end{array}$ & $\begin{array}{l}\text { Os autores referem que a } \\
\text { frequência de polifarmácia } \\
\text { pode estar relacionada ao uso } \\
\text { exacerbado ou inapropriado } \\
\text { de medicamentos, sendo um } \\
\text { desafio para a garantia de uma } \\
\text { assistência apropriada e segura } \\
\text { quanto ao processo de utilização } \\
\text { de medicamentos na APS }\end{array}$ \\
\hline
\end{tabular}




\section{DISCUSSÃO}

Segurança do paciente é uma temática cada vez mais discutida e, apesar de uma boa proporção da assistência à saúde no Brasil ser realizada no âmbito da APS, a maioria dos estudos relacionados a esse tema é realizada no contexto hospitalar, aspecto que é compreensível, tendo em vista que é nele que são realizados serviços mais complexos e com maior base tecnológica ${ }^{(9,23)}$.

Entretanto, a abordagem da temática também é essencial no contexto da APS ${ }^{(23,33-34)}$. Em 2008, em revisão publicada pela OMS, foi evidenciado que, apesar de haver muitos trabalhos e pesquisas interessantes do ponto de vista relacionado à segurança do paciente na APS, a discussão desse tema ainda estava incipiente à época, havendo necessidade de mais estudos e com base metodológica consistente com objetivo de viabilizar comparação entre resultados ${ }^{(4)}$.

Uma limitação importante no estudo acima referido esteve relacionada à língua: somente estudos na língua inglesa foram considerados ${ }^{(4)}$. No entanto, cabe referir que conclusão semelhante quanto à necessidade de mais estudos na área foi evidenciada por Marchon \& Mendes Junior (20l4) em revisão sistemática que ampliou o critério de inclusão para as línguas espanhola e portuguesa ${ }^{(9)}$. No entanto, na amostragem final não houve artigos sobre o tema em países em desenvolvimento, como o Brasil(9).

Aspecto importante evidenciado nas duas revisões acima mencionadas é o referente ao fato de que, dentre os tipos de incidentes mais encontrados na APS estão os associados ao processo de uso de medicamentos, desde a prescrição até seu uso pelo paciente ${ }^{(7-9)}$.

Destarte, a singularidade de cada prescrição deve ser considerada, tendo em vista a polifarmácia como um fator contribuinte para a ocorrência de eventos adversos, estando a toda a equipe interdisciplinar diretamente ligada à estas ações de vigilância essenciais para a manutenção da segurança do paciente neste âmbito. Sendo assim, todos os membros da equipe tornam-se coautores principais e, logo, corresponsáveis, nos processos preventivos de eventos adversos relacionados ao uso de medicamentos ${ }^{(35)}$.

Com base no recorte temporal da presente pesquisa, diferentemente do evidenciado na revisão sistemática publicada em $2014^{(9)}$ foram encontrados seis artigos publicados no Brasil com a temática relacionada ao uso de medicamentos na APS considerando sua vertente quanto à segurança do paciente. Alguns aspectos podem justificar ○ fato de a presente revisão ter evidenciado tais estudos e a revisão de 2014 não, como, por exemplo, diferença quanto aos descritores utilizados, bases de dados diferentes (nessa revisão integrativa, a base do Web of Science foi considerada, por exemplo), além da questão temporal (a revisão sistemática publicada em 2014 considerou trabalhos publicados até 2012). Fatores como a inclusão de artigos na amostra a partir do ponto de vista dos autores também devem ser considerados.

Apesar de terem sido evidenciados mais trabalhos em comparação à revisão publicada em 20I4, refletindo uma maior discussão acerca da temática no Brasil, ainda cabe a menção de que a proporção da abordagem é pequena diante da sua importância clínica e assistencial especialmente se considerado o objetivo de se discutir de forma mais específica segurança quanto ao processo complexo de uso de medicamentos na APS no Brasil. Além disso, cabe ressaltar a necessidade da discussão do papel da equipe interdisciplinar nesse contexto, aspecto que ainda corresponde a um desafio ao cuidado integral ao paciente, especialmente no que se refere ao uso seguro de tecnologias em saúde ${ }^{(7,10,13,22)}$.

Somente um estudo da amostra dessa revisão foi do tipo prospectivo e buscou de maneira longitudinal avaliar a gravidade dos eventos adversos e seus fatores contribuintes (nível de evidência mais forte - nível 3) ${ }^{(30)}$. Nesse estudo foram evidenciados problemas relacionados ao tratamento dos pacientes (principalmente erro de prescrição) e a aspectos administrativos, como, por exemplo, questões logísticas relacionadas ao abastecimento de medicamentos e produtos para saúde. Tais problemas culminaram em incidentes com importantes consequências clínicas: muitos resultaram em danos ao paciente, boa parte com gravidade muito alta, como dano permanente ou óbito( ${ }^{(30)}$.

Os demais estudos eram do tipo transversal, com menor nível de evidência associado (nível 6) e, apesar de tratarem aspectos como IMP, polifarmácia e medicamentos com risco importante associado ao seu uso, tiveram pouca abordagem para além do levantamento de frequências, com foco na prescrição de medicamentos $^{(27-29,31-32)}$. Nesses cinco estudos transversais, as elevadas frequências de IMP e/ou de polifarmácia chamam atenção e, apesar de não apresentarem correlação dos dados evidenciados com incidentes/eventos adversos por conta de suas limitações metodológicas, tais estudos também são importantes do ponto de vista científico no

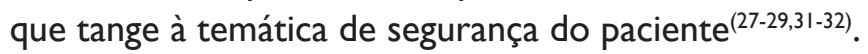

Considerando o processo de transição epidemiológica com o aumento da expectativa de vida e da prevalência e incidência de doenças crônicas, além da ampliação da disponibilidade e acesso de intervenções farmacológicas, a perspectiva acerca do uso de múltiplos medicamentos na assistência à saúde tem mudado, com subsequente aumento do risco de IMP e outros eventos adversos. Desse modo, é imprescindível que todo o processo relacionado ao uso dessas intervenções deve ser racionalizado, desde sua prescrição até seu uso, perpassando necessariamen- 
te pelo aprimoramento das práticas interdisciplinares relacionadas, com o objetivo de realização de atividades seguras e, como consequência, redução da probabilidade de eventos adversos ${ }^{(10,32)}$.

Dos cinco estudos de desenho transversal, somente um teve referência clara à análise de risco associado ao uso de alguns medicamentos de forma específica (como, por exemplo, aqueles com maior risco de ocasionar queda e complicação renal, medicamentos de baixa margem terapêutica e ditos inapropriados para idosos ${ }^{(31)}$. No entanto, não houve menção aos Medicamentos Potencialmente Perigosos (MPP), amplamente discutidos no que se refere à segurança no processo de utilização de medicamentos e caracterizados como de maior risco de trazer danos graves aos pacientes e que, a partir de prerrogativas da OMS, devem ser devidamente identificados, além de terem claras e evidentes à equipe e ao paciente a indicação, efeitos adversos potenciais e orientação quanto ao uso.Via de regra as equipes de farmácia e enfermagem destacam-se no processo de gestão quanto aos MPP com ações que variam desde a educação permanente à saúde até a própria investigação/notificação dos eventos adversos relacionados ${ }^{(36-37)}$.

Um aspecto positivo quanto aos estudos da amostra final foi que todos eles, mesmo que em diferentes proporções, indicaram sugestões para viabilizar aspectos assistenciais mais seguros relacionados ao uso de medicamentos, desde serviços farmacêuticos técnico-gerencias, como os vinculados à seleção de medicamentos e subsequente definição de uma lista de medicamentos seguros e com qualidade passíveis de prescrição e dispensação(29), até serviços farmacêuticos técnico-assistenciais, como análise de prescrição e atividades de farmácia clínica ${ }^{(28,32)}$, atividades de reconciliação medicamentosa quando da transição do paciente por níveis de atenção até a APS com apoio de toda a equipe interdisciplinar ${ }^{(31)}$ e atividades e educação em saúde tanto para pacientes quanto para a equipe envolvida nas atividades ${ }^{(27)}$.

É importante referir que gerenciamento de risco, planejamento (envolvendo atividades gerenciais e assistenciais), implementação de protocolos, ajustes de condições de trabalho (em termos estruturais e ambientais), comunicação efetiva e atividades ditas de práticas avançadas, especialmente em relação à enfermagem, além dos aspectos mencionados anteriormente, também podem auxiliar a implementação e/ ou aperfeiçoar a cultura de segurança na APS sob a perspectiva de aprimoramento organizacional de um cuidado seguro relacionado à prevenção de incidentes ${ }^{(7,13,20,23,38-39)}$.

Apesar da abordagem relacionada à segurança do paciente na APS ainda ser incipiente, há vários métodos disponíveis na literatura para avaliação dos incidentes relacionados ao cuidado em saúde na atenção primária à saúde ${ }^{(40)}$, sendo necessária para tal a incorporação de ferramentas que embasem o monitoramento de condições consideradas geradoras de risco à ocorrência de eventos adversos $^{(41)}$. Essa variedade metodológica foi evidenciada na presente revisão, já que não houve um padrão metodológico quanto à coleta e análise de dados no que se refere ao processo seguro de uso de medicamentos, algo trazido como essencial para investigação de incidentes no âmbito da APS com vistas a facilitar a mensuração de incidentes nesse contexto e, consequentemente, viabilizar comparações entre estudos ${ }^{(40)}$.

O estudo de Marchon, Mendes Junior \& Pavão (2015) foi o único em que houve aplicação de um instrumento de coleta de dados específico validado para profissionais de saúde visando avaliar se houve algum incidente durante o cuidado e quais suas características em termos de gravidade e fatores que contribuíram para sua ocorrência ${ }^{(30)}$. Trata-se de um questionário baseado no estudo australiano Primary Care International Study of Medical Errors (PCISME) ${ }^{(42)}$ o qual foi traduzido e adaptado à realidade brasileira ainda em $2015^{(40)}$ e que é sugerido pelos autores como alternativa para viabilização de estudos para obtenção de informações específicas na APS, de modo a favorecer comparações entre dados e fortalecer iniciativas nacionais e intervenções racionalizadas para a melhoria da segurança do paciente.

É importante referir algumas limitações dessa revisão integrativa, como a possibilidade de não inserção de alguma publicação relacionada à temática devido aos descritores; além disso, a utilização destes descritores em inglês possivelmente remeteu a publicações que os utilizaram nessa língua, o que se configura também em limitação do estudo, tendo em vista que mais estudos em português eram esperados.A possibilidade do viés de publicação deve ser referida, e, de forma mais específica, limitações referentes aos critérios de exclusão previstos na metodologia também devem ser consideradas. Outra limitação poderia estar associada à dificuldade de acesso a algumas publicações ditas restritas não disponibilizadas na íntegra. Além disso, a literatura cinzenta não foi considerada, a qual poderia viabilizar a leitura de mais pesquisas vinculadas à temática.

Ressalta-se que a segurança do paciente na APS deve ser incentivada, aprimorada e avaliada constantemente pela equipe interdisciplinar, gestores e usuários a fim de otimizar a assistência prestada, com ênfase na vigilância de riscos que, rotineiramente, não são tão discutidos e questionados como no contexto da atenção secundária e terciária, mas, que fazem parte da assistência primária e que possibilitam inúmeras variações da efetividade do gerenciamento e do cuidado em saúde. Nesse sentido, como contributo, a presente revisão evidencia e certifica a necessidade de produção científica, especialmente 
interdisciplinar, relacionada à segurança do paciente na APS já referida em estudos anteriores, especialmente em relação ao uso de medicamentos, embora fique registrada a relevância das pesquisas cujos dados e abordagem foram compilados nessa revisão.

\section{CONCLUSÃO}

Considerando a importância da discussão da temática de uso de medicamentos no tocante à necessidade que seja um processo seguro e com qualidade, poucos artigos constaram na amostra final na revisão proposta, o que evidencia e certifica a escassez de estudos com a temática de avaliação nesse contexto também mencionada em estudos anteriores.

Apesar de serem observadas sugestões importantes com objetivo de melhorar as atividades relacionadas ao processo de utilização dos medicamentos sob o ponto de vista de segurança do paciente, verificou-se uma divergência metodológica associada aos estudos da amostra, evidenciando a necessidade de uma padronização relacionada com vistas a facilitar a obtenção de informações e, a longo prazo, viabilizar intervenções racionais associadas a esse processo.

Essa escassez de pesquisas e o foco em levantamento de frequências de problemas como IMP e polifarmácia sem ampla discussão acerca de seus impactos em termos de incidência de eventos adversos nas publicações brasileiras, apontam para a necessidade de realização de mais pesquisas, especialmente longitudinais, que de fato remetam à discussão da gestão de risco associado ao processo de utilização de medicamentos no âmbito da APS. Tais pesquisas subsidiarão o diagnóstico dos serviços em relação à temática de segurança no uso de medicamentos e, consequentemente, auxiliarão no processo de tomada de decisão e a formulação de estratégias envolvendo toda a equipe interdisciplinar que visem ampliar a capacidade de gestão local com o objetivo de realização de atividades qualificadas e seguras com subsequente redução da probabilidade de eventos adversos associados. 


\section{REFERÊNCIAS}

I. Mendes EV.As redes de atenção à saúde. Ciênc Saúde Coletiva. 20 I0; I5(5):2297-2305. doi: http://dx.doi.org/I0.1590/ SI4I3-8I232010000500005

2. Figueira MCS, Silva WP, Silva EM. Integrative literature review: access to primary healthcare services. Rev Bras Enferm [Internet]. 2018;7I(3):II78-88. doi: http://dx.doi. org/| 0.1590/0034-7|67-2017-044|

3. Barreto ACO, Rebouças CBA, Aguiar MIF, Barbosa RB, Rocha SR, Cordeiro, LM, et al. Perception of the Primary Care multiprofessional team on health education. Rev Bras Enferm [Internet]. 2019;72(Suppl I):266-73. doi: http://dx.doi. org/l 0.1590/0034-7I67-2017-0702

4. Makeham MAB, Dovey SM, Runciman W, Larizgoitia I. Methods and Measures used in Primary Care Patient Safety Research: Results of a literature review. World Organization of Health [Internet]. 2008 [cited Jun 03].Available from: https://www.who.int/patientsafety/research/methods_measures/makeham_dovey_full.pdf.

5. Sousa P, Mendes Junior WV. Segurança do paciente: conhecendo os riscos nas organizações de saúde. Rio de Janeiro: Fiocruz; 20l4. 45 Ip.

6. Forte ECN, Pires DEP, Martins MMFPS, Padilha MICS, Schneider DG, Trindade LL. Nursing errors in the media: patient safety in the window. Rev Bras Enferm [Internet]. 2019;72(Suppl I):189-96. doi: http://dx.doi. org/I0.1590/0034-7I67-2018-01 I3

7. Souza MM, Ongaro JD, Lanes TC, Andolhe R, Kolankiewicz ACB, Magnago TSBS. Patient safety culture in the Primary Health Care. Rev Bras Enferm [Internet]. 201 9;72(I):27-34. doi: http://dx.doi.org//0.1590/0034-7/67-2017-0647

8. Ministério da Saúde (BR), Agência Nacional de Vigilância Sanitária (ANVISA). Protocolo de Segurança na Prescrição, Uso e administração de medicamentos [Internet]. Brasília: Ministério da Saúde/ ANVISA; 2013 [cited Sep 02].Available from: https://www20.anvisa.gov.br/segurancadopaciente/ index.php/publicacoes/item/seguranca-na-prescricao-uso-e -administracao-de-medicamentos.

9. Marchon SG, Mendes Junior WV. Patient safety in primary health care: a systematic review. Cad Saúde Pública. 20|4;30(9):18I5-35. doi: http://dx.doi.org//0.1590/ $0102-3$ I IX00II4II3

10. Mieiro DB, Oliveira EBC, Fonseca REP, Mininel VA, Zem -Mascarenhas SH, Machado RC. Strategies to minimize medication errors in emergency units: an integrative review. Rev Bras Enferm [Internet]. 2019;72(Suppl I):307-I4. Doi: http://dx.doi.org//0.1590/0034-7/67-2017-0658

I I. Ministério da Saúde (BR), Fundação Oswaldo Cruz,Agência Nacional de Vigilância Sanitária. Documento de referência para o Programa Nacional de Segurança do Paciente [Internet]. Brasília: Ministério da Saúde; 2014 [cited 2019 Aug 20]. Available from: http://bvsms.saude.gov.br/bvs/publicacoes/ documento_referencia_programa_nacional_seguranca.pdf.
12. Macedo RS, Bohomol E. Validation of self-assessment instrument for the Patient Safety Center. Rev Bras Enferm [Internet]. 2019;72(Suppl I):259-65. doi: http://dx.doi. org/l0.1590/0034-7I67-2017-0657

13. Magalhães AMM, Kreling A, Chaves EHB, Pasin SS, Castilho BM. Medication administration - nursing workload and patient safety in clinical wards. Rev Bras Enferm [Internet]. 2019;72(I):183-9. doi: http://dx.doi.org/10.1590/00347167-2018-0618

14. World Organization of Health [Internet]. Geneva:WHO; 2019a [cited 2019 Set 02]. Medication Safety in Polypharmacy. Available from: https:/apps.who.int/iris/bitstream/ handle/ I 0665/325454/WHO-UHC-SDS-20 I 9. I I-eng.pdf?ua=I.

I5. Mendes VLPS, Luedy A, Tahara ATS, Silva GTR. Política de qualidade, acreditação e segurança do paciente em debate. Rev Baiana Saúde Pública. 2017; 40: 232-249. doi: http://dx. doi.org/ 0.22278/23 I8-2660.2016.v40.n0.a2678

16. Siman AG, Cunha GSS, Brito MJM.The practice of reporting adverse events in a teaching hospital. Rev Esc Enferm USP. 20 I7;5 I:e03243:I-8. doi: http://dx.doi.org/I0.1590/s I980$220 \times 2016045503243$

17. Báo ACP, Amestoy SC, Moura GMSS, Trindade LL. Quality indicators: tools for the management of best practices in Health. Rev Bras Enferm. 2019;72(2):360-6. doi: http://dx. doi.org/10.1590/0034-7/67-2018-0479

18. Cavalcante EFO, Pereira IRBO, Leite MJVF, Santos AMD, Cavalcante CAA. Implementação dos núcleos de segurança do paciente e as infecções relacionadas à assistência à saúde. Rev Gaúcha Enferm. 2019;40(esp):e20180306. doi: https://doi.org/10.1590//983- I447.2019.20180306

19. Siman AG, Cunha SGS, Brito MJM. Nursing actions for patient safety in hospitals: integrative review. Rev Enferm UFPE on line. 2017;II(2):1016-24. doi: https://doi.org/ | 0.5205/ I 98I-8963-v I Ii2a I 3472p I 0 I6-I024-20 I7

20. Carvalho PA, Gottems LBD, Motta LACR, Laundos CAS, Juliano JVS. Assessment of safety culture in a public hospital in the Federal District, Brazil. Rev Bras Enferm [Internet]. 2019;72(Suppl I):252-8. doi: http://dx.doi. org/10.1590/0034-7167-2017-07/6

21. Magalhães FHL, Pereira ICA, Luiz RB, Barbosa MH, Ferreira MBG. Patient safety atmosphere in a teaching hospital. Rev Gaúcha Enferm. 2019;40(esp):e20180272. doi: https://doi. org/10.1590/1983-1447.2019.20180272

22. Peruzzo HE, Silva ES, Batista VC, Haddad MCFL, Peres AM, Marcon SS. Organizational climate and teamwork at the Family Health Strategy. Rev Bras Enferm. 2019;72(3):721-7. doi: http://dx.doi.org/10.1590/0034-7/67-2017-0770

23. Raimondi DC, Bernal SCZ, Oliveira JLC, Matsuda LS. Cultura de segurança do paciente na atenção primária à saúde: análise por categorias profissionais. Rev Gaúcha Enferm. 2019;40(esp):e20180 I33. doi:https://doi.org/10.1590//983|447.20|9.20|80|33 
24. Souza MT, Silva MD, Carvalho R. Integrative review: what is it? How to do it?. Einstein. 2010;8(I): 102-6. doi: http:// dx.doi.org/ | 0.1590/s I679-450820 I Orw I I 34

25. Mendes KDS, Silveira RCCP, Galvão CM. Integrative literature review: a research method to incorporate evidence in health care and nursing. Texto Contexto Enferm. 2008; I7(4):758-64. doi: http://dx.doi.org/I0.I590/S0I0407072008000400018

26. Melnyk B, Fineout-Overholt E. Evidence-based practice in nursing and healthcare: a guide to best practice. Philadelphia:Wolters Kluwer; 201 I. 599p.

27. Nicolini P, Nascimento JWL, Greco KV, Menezes FG. Fatores relacionados à prescrição médica de antibióticos em farmácia pública da região Oeste da cidade de São Paulo. Ciênc Saúde Coletiva. 2008; I3(Sup): 689-96. doi: http://dx. doi.org/I0.I590/SI4|3-8I2320080007000I8

28. Mendonca JMD, Lyra Junior DP, Rabelo JS, Siqueira JS, Balisa-Rocha BJ, Gimenes FRE, et al. Analysis and detection of dental prescribing errors at Primary Health Care Units in Brazil. Pharm World Sci. 20 I0; 32(I):30-5. doi: http://dx.doi. org/ I0.1007/s I 1096-009-9335-7

29. Teixeira JJV, Crozatti MTL, Dos Santos CA, Romano-Lieber NS. Potential Drug-Drug Interactions in Prescriptions to Patients over 45 Years of Age in Primary Care, Southern Brazil. PLoS ONE. 20I2;7(I0): I-6. doi: http://dx.doi. org/10.137//journal.pone.0047062

30. Marchon SG, Mendes Junior WV, Pavão AL. Characteristics of adverse events in primary health care in Brazil. Cad. Saúde Pública. 20 I 5;3 I ( I I): I - I6. doi: http://dx.doi.org/ I0.1590/ 0102-3।IX00194214

31. Cruz HL, Mota FKC, Araújo LU, Bodevan EC, Seixas SRS, Santos, DF. The utility of the records medical: factors associated with the medication errors in chronic disease. Rev Lat Am Enfermagem. 2017; 25: e2967. doi: https://doi. org/I0.1590/I5I8-8345.2406.2967

32. Nascimento RCRM, Álvares J, Guerra Junior AA, Gomes IC, Silveira MR, Costa EA, et al. Polypharmacy: a challenge for the primary health care of the Brazilian Unified Health System. Rev Saude Publica. 2017; 5 I Supl 2:19s. doi: http:// dx.doi.org/10.1 |606/s I518-8787.2017051007|36

33. World Organization of Health [Internet]. Geneva:WHO; 2019b [cited 2019 Set 02]. Patient safety: safer primary care. Available from: http://www.who.int/patientsafety/safer_primary_care/en/.
34. Silva APF, Backes DS, Magnago TSBS, Colomé JS. Patient safety in primary care: conceptions of family health strategy nurses. Rev Gaúcha Enferm. 2019;40(esp):e20180164. doi: https://doi.org/10.1590/1983- 1447.2019.20180164

35. Moreira MB, Mesquita MGR, Stipp MAC, Paes GO. Potential intravenous drug interactions in intensive care. Rev Esc Enferm USP. 20I7;5I:e03233. doi: http://dx.doi.org/I0.1590/ S1980-220X2016034803233

36. Reis GAX, Hayakawa LY, Murassaki ACY, Matsuda LM, Gabriel CS, Oliveira MLF. Nurse manager perceptions of patient safety strategy implementation. Texto Contexto Enferm [Internet], 2017; 26(2):e003400 I6. doi: http://dx.doi. org/I0.1590/0104-07072017000340016

37. Reis MAS, Gabriel CS, Zanetti ACB, Bernardes A, Laus AM, Pereira LRL. Potentially hazardous drugs: identification of risks and error prevention barriers in intensive care. Texto Contexto Enferm [Internet], 2018; 27(2):e57/0016. doi: http://dx.doi.org/10.1590/010407072018005710016

38. Figueiredo ML, Oliveira e Silva CS, Brito MFSF, D’Innocenzo M. Analysis of incidents notified in a general hospital. Rev Bras Enferm [Internet]. 20 I8;7 I ( ): I I I-9. doi: http://dx.doi. org/I0.1590/0034-7/67-2016-0574

39. Amaral LR, Araújo CA. Advanced practices and patient safety: an integrative literature review. Acta Paul Enferm. 2018;3।(6):688-95. doi: http://dx.doi.org/10.1590//9820194201800094

40. Marchon SG, Mendes Junior WV. Tradução e adaptação de um questionário elaborado para avaliar a segurança do paciente na atenção primária em saúde. Cad Saúde Pública. 20I5;3I(7):I395-I402. doi: http://dx.doi.org/I0.1590/ $0102-311 \times 00157214$

4I.Araújo PR, Lima FET, Ferreira MKM, Oliveira SKP, Carvalho REFL, Almeida PC. Medication administration safety assessment tool: construction and validation. Rev Bras Enferm. 2019;72(2):329-36. doi: http://dx.doi.org/10.1590/00347I67-2018-0340

42. Makeham MAB, Dovey SM, County M, Kidd MR. An international taxonomy for errors in general practice: a pilot study. Med J Aust. 2002; I5(I77): 68-72. doi: https://doi.org/I0.5694/j.I326-5377.2002.tb04668.x

Recebido: 2020-03-12

Aceito: 2020-04-09 